(2) Open Access Full Text Article

REVIEW

\title{
Clinical potential of mechlorethamine gel for the topical treatment of mycosis fungoides-type cutaneous T-cell lymphoma: a review on current efficacy and safety data
}

This article was published in the following Dove Press journal:

Drug Design, Development and Therapy

\author{
Kendall Liner ${ }^{1}$ \\ Celeste Brown ${ }^{2}$ \\ Laura Y McGirt ${ }^{3}$ \\ 'Division of Dermatology, Medical \\ College of Georgia at Augusta \\ Health, Augusta, GA, USA; ${ }^{2}$ School \\ of Medicine, University of North \\ Carolina at Chapel Hill, Carolinas \\ Medical Center, Charlotte, NC, \\ USA; ${ }^{3}$ Department of Hematology/ \\ Oncology, Levine Cancer Institute, \\ Carolinas Medical Center. \\ Charlotte, NC, USA
}

\begin{abstract}
Nitrogen mustard is a chemotherapeutic agent that has a well-documented safety and efficacy profile in the treatment of cutaneous T-cell lymphoma. Development of nitrogen mustard formulations and treatment regimens has been studied extensively over the last 40 years. In the last 5 years, a new gel formulation has been developed that is associated with a decrease in delayed hypersensitivity reactions. The authors in this review found that while the gel formulation may result in a decrease of allergic contact dermatitis, this advantage has been replaced by a higher number of irritant contact reactions and a decrease in complete response rate. The gel formulation has a complete response rate of $13.8 \%$, which is a decrease in efficacy when compared to aqueous-based preparations of similar concentrations.
\end{abstract}

Keywords: mycosis fungoides, nitrogen mustard, mechlorethamine gel, cutaneous T-cell lymphoma, CTCL, Valchlor ${ }^{\circledR}$

\section{Introduction}

Mycosis fungoides (MF) is the most common type of cutaneous T-cell lymphoma (CTCL) and is characterized by its progression from patch to plaque to tumor stage of disease. Clinically, patients will typically have several years of nonspecific skin changes that may resemble psoriasis or eczema and often may have multiple previous nondiagnostic biopsies. Histologically, MF consists primarily of an epidermotropic dermatitis with an atypical lymphocytic infiltrate, and characteristic Pautrier microabscesses. ${ }^{1}$

Treatment of MF is highly dependent upon the stage of disease. The current staging system (Table 1) is based on the new MF and Sézary Syndrome criteria proposed by Olsen et al. It is a tumor, node, metastasis, blood (TNMB) classification that takes into account the type and surface area of skin lesions in addition to lymph node, visceral, and circulating blood lymphocytes. ${ }^{2}$

Mechlorethamine or nitrogen mustard (NM) has a primary role in the early stages of the skin-limited disease and has been studied for its efficacy in MF since $1959 .{ }^{3}$ NM is a topical chemotherapeutic alkylating agent that affects rapidly dividing cells. Cytotoxicity to DNA is postulated to occur by DNA cross-linking, nucleic acid depurination, or abnormal base paring. Additionally, it may alter the growth pattern of the tumor and enhance immunogenic host potential..$^{4,5}$ Most data in regard to the safety and efficacy of topical NM involve Stage IA to Stage III, with the majority
Correspondence: Laura Y McGirt Levine Cancer Institute, Ste 300, I02I Morehead Medical Drive, Suite A, Charlotte, NC 28204, USA

Tel +l 9804426367

Fax + I 9804426321

Email laura.y.mcgirt@

carolinashealthcare.org 
Table I Staging criteria for cutaneous T-cell lymphoma

\begin{tabular}{|c|c|c|c|c|c|}
\hline Stage & Clinical description & Tumor (T) & Node (N) & Metastasis (M) & Blood (B) \\
\hline IA & Limited patch/plaque $<10 \%$ & $\mathrm{TI}$ & No & Mo & $0-1$ \\
\hline IB & Generalized patch/plaque $>10 \%$ & $\mathrm{~T} 2$ & No & Mo & $0-1$ \\
\hline IIA & Patch/plaque + adenopathy & $\mathrm{TI}-2$ & $\mathrm{NI}-2$ & Mo & $0-1$ \\
\hline IIB & Tumors \pm adenopathy & T3 & No-2 & Mo & $0-1$ \\
\hline III & Erythroderma \pm adenopathy & $\mathrm{T} 4$ & No-2 & Mo & $0-1$ \\
\hline IVA & Histologically + nodes or Sézary syndrome & $\mathrm{TI}-4$ & No-3 & Mo & $0-2$ \\
\hline IVB & Visceral involvement & $\mathrm{TI}-4$ & No-3 & MI & $0-2$ \\
\hline
\end{tabular}

Notes: Adapted from Olsen E, Vonderheid E, Pimpinelli N, et al; ISCL/EORTC. Revisions to the staging and classification of mycosis fungoides and Sezary syndrome: a proposal of the International Society for Cutaneous Lymphomas (ISCL) and the cutaneous lymphoma task force of the European Organization of Research and Treatment of Cancer (EORTC). Blood. 2007; I 10(6): 1713-1722. @ 2007 by The American Society of Hematology. ${ }^{2}$

of studies involving between Stage IA and Stage IIA. Over the last 30 years, treatment of MF with NM has evolved in regard to site of application (whole body versus spot treatment), vehicle method (aqueous, ointment, versus gel), concentration, and duration of treatment. Several studies have documented $51 \%-84 \%$ complete response (CR) for patients in the T1 stage (limited patch/plaque) and 31\%-62.2\% CR for patients in the T2 stage (generalized patch/plaque) of disease. ${ }^{6-10}$ While efficacy is well established, the adverse effects of topical treatment with aqueous- and ointment-based NM have also been well described. These include the more common allergic contact dermatitis (ACD)/delayed type hypersensitivity reactions (DHRs) as well as the immediate urticarial and irritant type reactions. The new gel formulation is associated with irritation, hyperpigmentation, pruritus, erythema, and contact dermatitis. ${ }^{11,12}$ Additionally, other studies have both refuted and documented a change in incidence of secondary skin cancers or systemic manifestations of therapy with topical NM. ${ }^{4,6-8,11,13}$ This article serves to provide a comprehensive review of both the safety and efficacy of mechlorethamine gel compared to other NM formulations in treatment of the early stages of the MF-type of CTCL.

\section{Materials and methods}

A comprehensive literature search was performed using the PubMed database. The following search terms were used: NM or mechlorethamine and mycosis fungoides (and safety), NM or mechlorethamine and mycosis fungoides (and efficacy), and NM or mechlorethamine and gel. Selected publications for review included cohort studies, prospective and retrospective studies, review articles, and randomized controlled trials.

\section{NM}

The first preparations of topical NM or mechlorethamine therapy were aqueous-based solutions that consisted of $10-20 \mathrm{mg}$ of NM dissolved in $40-60 \mathrm{~mL}$ of water. ${ }^{4-6,8,9,14,15}$
Typical protocols in the late 1970 s and into the 2000s involved mixing the solution as above with application of the solution to the whole body with caution in the intertriginous areas. If the patient developed a contact or hypersensitivity reaction, reapplication was attempted with NM further diluted in water (1,200-1,800 mL), often after initial treatment cessation and addition of topical steroids. ${ }^{4,5}$ The duration and frequency of application of treatment are varied in the literature, but typically range initially from daily to twice weekly until $\mathrm{CR}$ is achieved followed by a maintenance regimen lasting 6-12 months. In 1973, Van Scott and Kalmanson reported the frequency of application from three times per week to twice daily with maintenance doses of once weekly after CR was obtained..$^{15}$ Other studies by Ramsay et al reported daily application until CR was achieved followed by continued daily use 6 months after, then biweekly use for another 6 months, then once weekly for the final 6 months. ${ }^{4}$ There have been additional investigations with different induction and maintenance regimens by Lindahl et al. These studies were based on daily application of NM for 2 weeks followed by subsequent treatments every fourth and eighth week until treatment was no longer indicated. ${ }^{10}$ Given the cutaneous hypersensitivity reactions, xerosis, and difficulty with application, ointments and gels have been developed to simplify application and improve adherence to medication use. Price et al first experimented with ointment-based preparations of NM, and this was also typically applied to the entire body surface. ${ }^{16}$ More recent studies with both ointment and gel preparations by Lessin et al focus on both spot treatment and total body treatment depending on the stage of the disease. ${ }^{11}$

\section{Efficacy}

The efficacy of NM in treating MF was established in the 1950 s as it became a topical treatment option in 1959 in the USA. ${ }^{3}$ Table 2 outlines the key prospective studies, retrospective studies, and clinical trials that documented the clinical response to NM as the primary endpoint. This table 


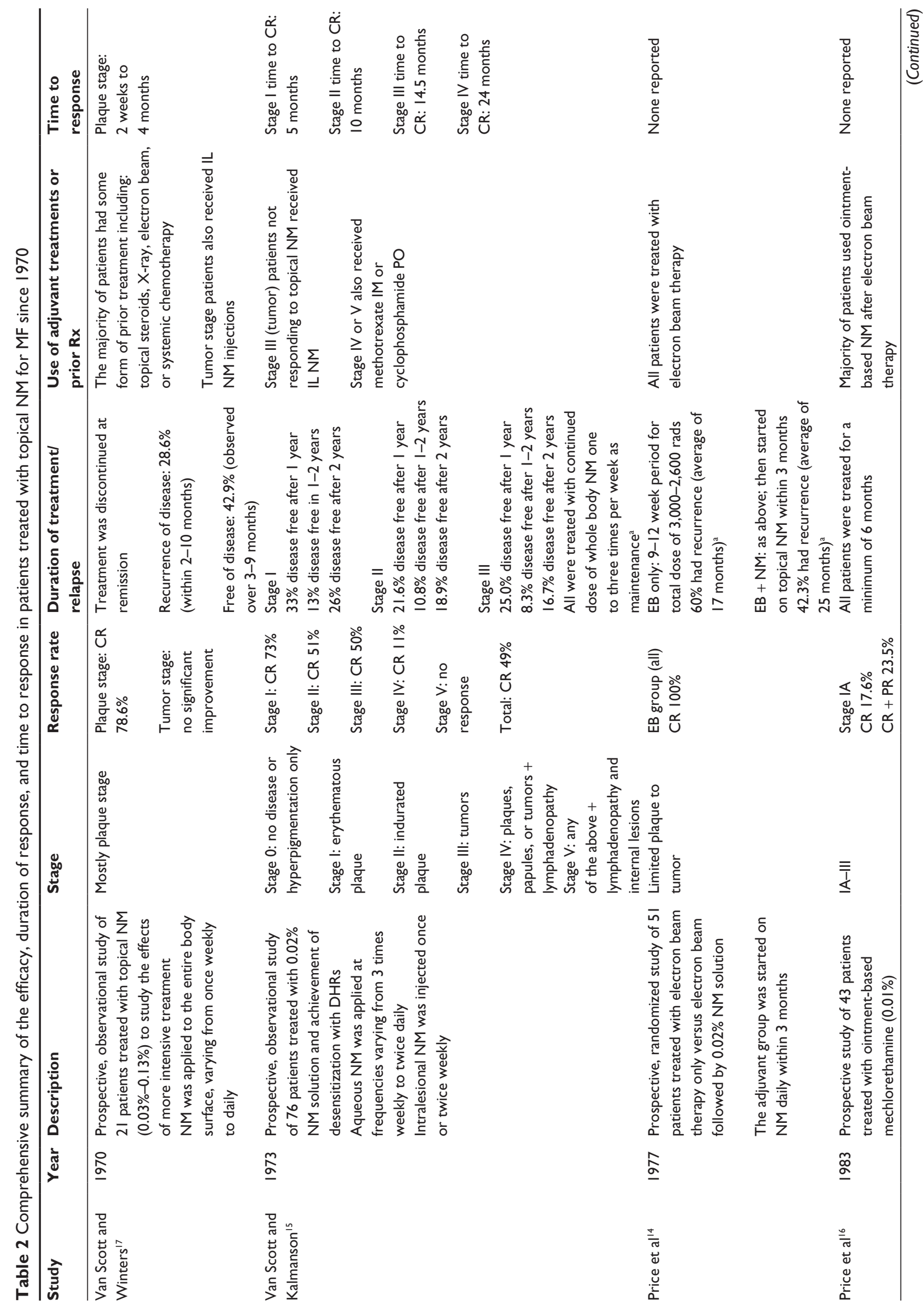




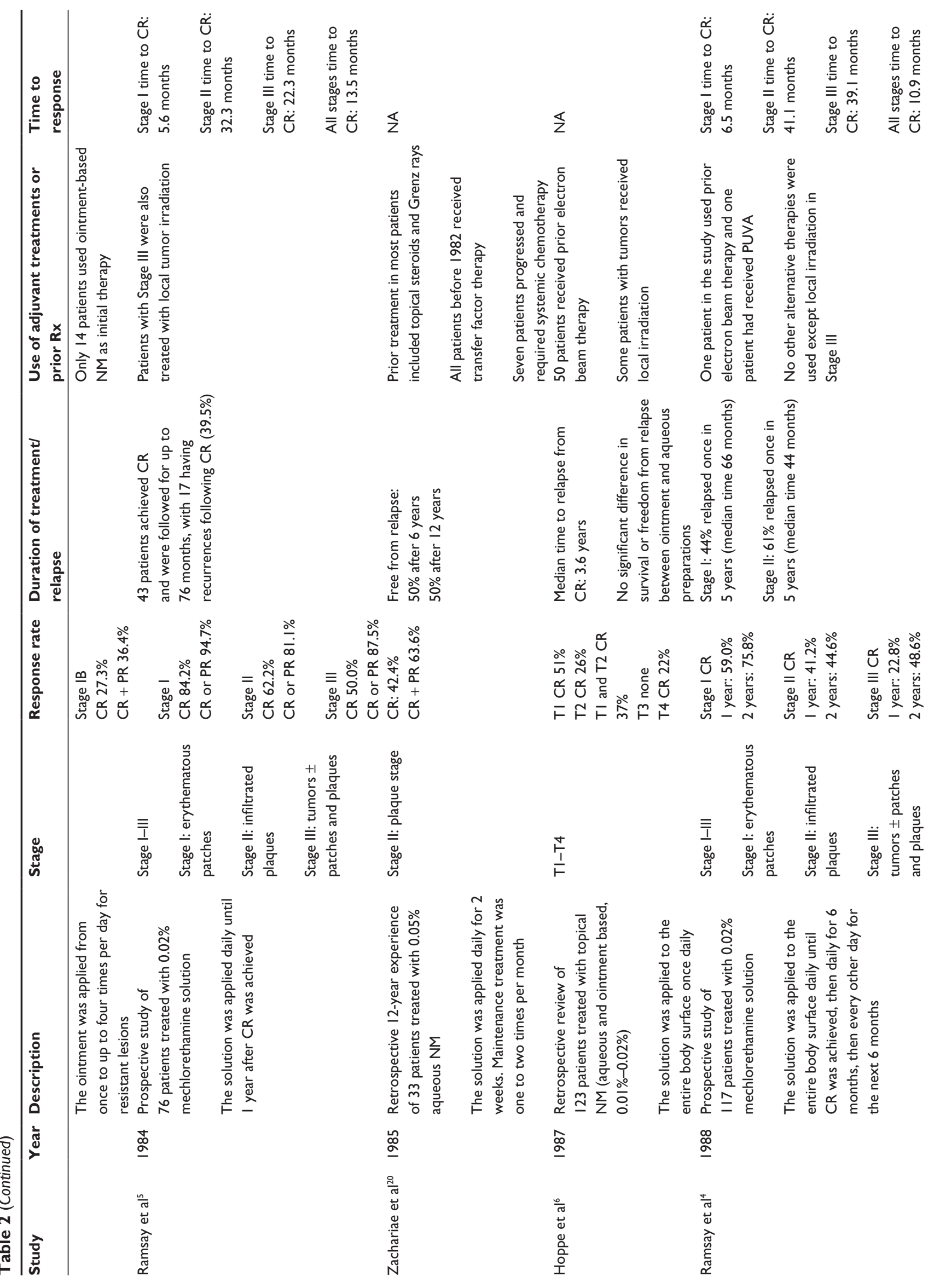




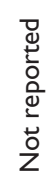
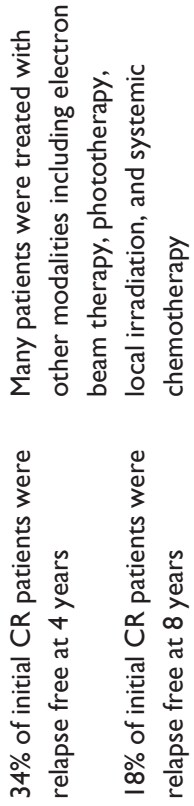

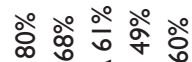

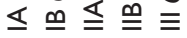

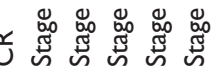
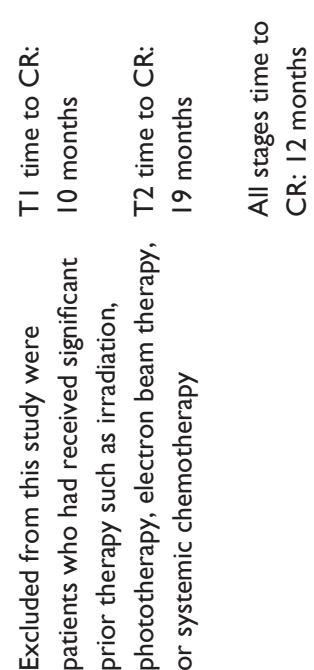
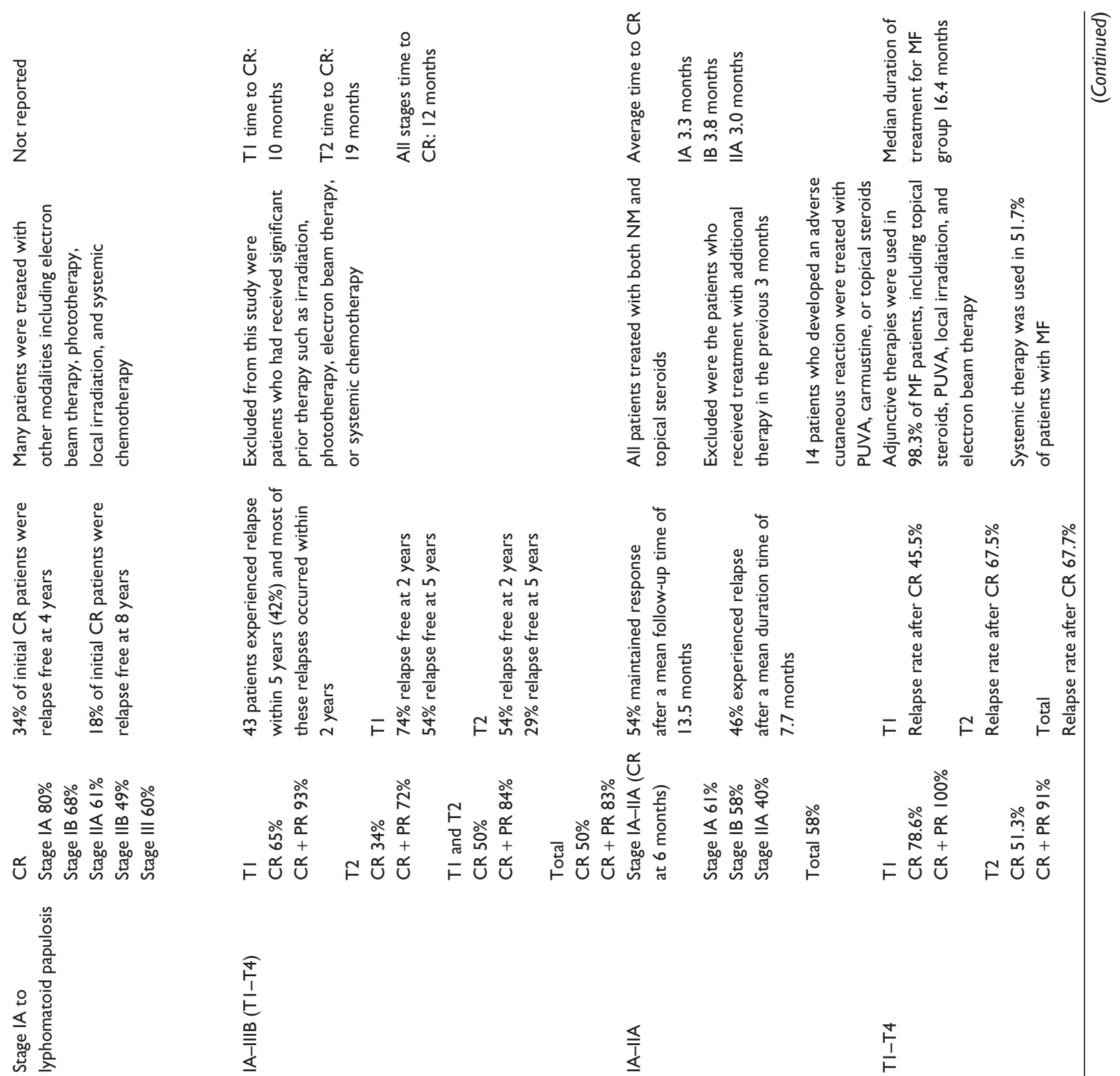
$\stackrel{\Upsilon}{\cup}$

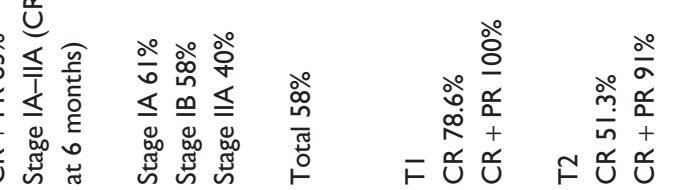

$\stackrel{F}{F}$
$\frac{1}{E}$
$\cong$
$\stackrel{\Xi}{\leq}$
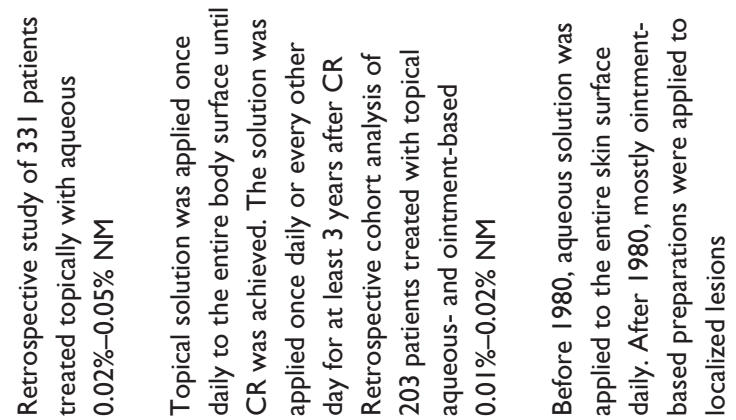

$\stackrel{\stackrel{\circ}{\circ}}{\stackrel{\circ}{\circ}}$

髉

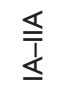

$\frac{⿱ 亠 䒑}{r}$
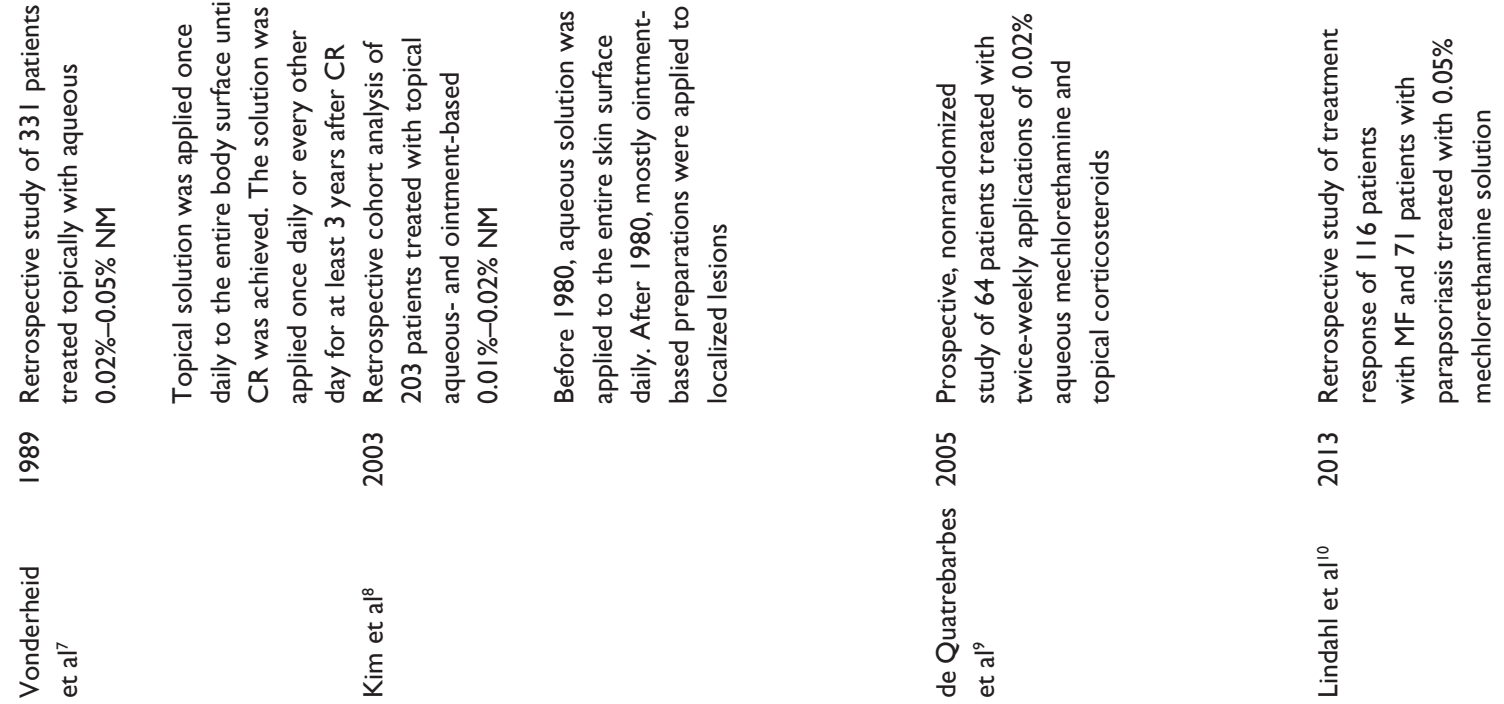

융

음

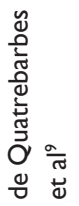

$\frac{0}{\pi}$
$\frac{0}{\frac{0}{5}}$
$\frac{\pi}{0}$
.$\frac{\pi}{7}$ 
includes studies with aqueous-, ointment-, and gel-based mechlorethamine preparations. Secondary endpoints typically included duration of treatment response, time to response or recurrence, and survival.

Van Scott and Winters ${ }^{17}$ and Price et a ${ }^{14,16}$ conducted several prospective, observational studies in the 1970s and 1980s regarding the efficacy of NM in treating MF. Van Scott and Winters in the 1970s found that higher concentrations of NM (10-40 mg of NM in 30-40 mL of water) resulted in CR of $78.6 \%$ of patients in the plaque stage of MF, but found no significant improvement in patients in the tumor stage of MF. They found that $28.6 \%$ of these patients experienced disease recurrence within 2-10 months, whereas $42.9 \%$ of patients remained free of disease. ${ }^{17}$ In 1973, Van Scott and Kalmanson further investigated the efficacy of aqueous and intralesional NM in patients with all stages of MF, and patients were followed for a minimum of 2 years and continued on maintenance doses of NM. Aqueous solutions were prepared at a concentration of $0.02 \%$ and applied twice daily to three times per week depending on the development of a DHR. Intralesional injections of $0.005 \% \mathrm{NM}$ were used once or twice per week to improve the response to topical NM in patients with tumors and refractory disease for an average of 2-3 weeks. In this study, staging guidelines were as follows: Stage I patients had erythematous plaques or generalized erythema; Stage II patients had indurated plaques with or without generalized erythema; and Stage III patients had tumors with or without plaques or generalized erythema. They found $50 \%-73 \%$ of patients with MF Stage I to Stage III were free of disease after 5-14.5 months. Stage I patients, on average, were free of disease in 5 months, compared to 10 and 14.5 months for stages II and III, respectively. After 2 years, $26 \%$ of Stage I patients, $18.9 \%$ of Stage II patients, and $16.7 \%$ of Stage III patients remained free of disease. ${ }^{15}$

Similar to NM, electron beam therapy was first established in the treatment of MF in the 1950s..$^{18}$ Price et al conducted a randomized, prospective study in 1977 to investigate treatment with adjuvant topical aqueous NM after electron beam therapy. They found that initial clinical response was $100 \%$ in all patients; however, patients in the electron beam only group had a recurrence of $60 \%$, compared to a recurrence of $42.3 \%$ in patients who received both electron beam treatment and adjuvant topical NM. ${ }^{19}$

Price et al were the first to prospectively study ointmentbased preparations of mechlorethamine in 1983. Ointmentbased preparations were developed primarily due to increasing reports of ACD with the aqueous-based preparations of NM. While the endpoint of this study was primarily to investigate 
the development of contact dermatitis with ointment-based preparations, CR after 6 months was documented as $17.6 \%$ for Stage IA (limited plaque $<10 \%$ ) patients and $27.3 \%$ for Stage IB (generalized plaque $>10 \%$ ) patients. The variation in the concentration of mechlorethamine in the ointment $(0.001 \%-0.02 \%)$ may account for the decrease in efficacy compared to other studies or a degree of patient nonadherence due to the greasiness of ointment-based preparations. ${ }^{16}$ Aqueous preparations in comparable studies typically were prepared at concentrations of $0.02 \%-0.05 \%$ mechlorethamine solution. ${ }^{4,5,10,15,19}$ The authors note that, theoretically, ointmentbased preparations should have better mass transfer through the skin's surface resulting in the use of lower amounts of mechlorethamine compared to aqueous-based solutions. ${ }^{16}$

During the mid to late 1980s, Ramsay et al conducted several prospective studies to further establish the efficacy of topical mechlorethamine solution. The study designs in both 1984 and 1988 included a larger patient population compared to the previously mentioned studies above and resulted in similar response rates. Staging for MF at the time of these studies was as follows: erythematous patches (Stage I), infiltrated plaques (Stage II), and tumors or ulcers (Stage III). In 1984, Ramsay et al evaluated 76 patients mostly in Stage I and Stage II treated with $0.02 \%$ mechlorethamine solution applied daily until 1 year after CR was achieved. CR was achieved in $84.2 \%$ and $62.2 \%$ of Stage I and Stage II patients, respectively, with an overall CR rate of $67.2 \%$ and an overall complete and partial response rate of $85.9 \%$. Average time to CR was comparable to earlier studies by Van Scott et al for Stage I and Stage III patients. The average time to CR was 5.6, 32.3, and 13.5 months for stages I, II, and III, respectively. It should be noted that average time to $\mathrm{CR}$ in Stage II patients is longer than Stage III patients in both the studies conducted by Ramsay et al in 1984 and 1988. Patients with Stage III disease were also treated with local irradiation of the tumor, which likely accounts for the shorter response time between the two stages. ${ }^{5}$ In 1988, Ramsay et al evaluated the efficacy of $0.02 \%$ mechlorethamine solution in 117 patients with early-stage MF after both 1 and 2 years of therapy. They reported a CR of $59 \%, 41.2 \%$, and $22.8 \%$ after 1 year for stages I, II, and III, respectively. A CR of $75.8 \%$, $44.6 \%$, and $48.6 \%$ was achieved after 2 years for stages I, II, and III, respectively. Average time to CR was 6.5, 41.1, and 39.1 months for stages I, II, and III, respectively. ${ }^{4}$

In 1985, Zachariae et al retrospectively studied the efficacy of aqueous-based topical NM in the plaque stage of MF. They found a CR of $42.4 \%$ and a complete and partial response of $63.6 \%$ in patients using topical NM to treat plaque stage MF (Stage II). ${ }^{20}$ Another larger retrospective study of 331 patients, completed by Vonderheid et al in 1989, found similar results with topical aqueous-based NM application in the early stages of MF. The concentration of NM applied to the entire skin surface was between $0.02 \%$ and $0.05 \%$. Stage IA patients achieved $80 \% \mathrm{CR}$, Stage IB patients achieved 68\% CR, Stage IIA achieved 61\% CR, Stage IIB achieved $49 \%$ CR, and $60 \%$ of Stage III patients achieved CR with daily use of NM until CR was achieved followed by maintenance dosing. Most of these patients were also receiving concurrent treatment with phototherapy or electron beam therapy. Approximately $34 \%$ of patients were relapse free at 4 years and $18 \%$ of patients were relapse free at 8 years. After 8 years, the authors observed no evidence of relapse in these patients. They concluded relapse is unlikely after remission for 8 or more years, supported by similar reports from Stanford University and Zachariae et al. 6,7,20

Stanford University School of Medicine (Kim et al) also retrospectively evaluated 203 patients with T1-T4 MF treated with aqueous- and ointment-based preparations (Aquaphor) of NM. Aqueous- and ointment-based concentrations were similar (10-20 mg of NM in $100 \mathrm{~mL}$ or $0.02 \%$ ). They found $\mathrm{CR}$ in $65 \%$ of patients with $\mathrm{T} 1$ after an average of 10 months and 34\% CR in patients with T2 after an average of 19 months. Seventy-four percent of patients in the T1 stage were relapse free at 2 years and $42 \%$ underwent relapse within 5 years. The survival of patients who were initially treated with topical NM was also evaluated. They found that $\mathrm{T} 1$ disease patients had a statistically significant improvement in disease-specific survival when compared to patients with T2 disease. Survival at 5 years was $97 \%$ for $\mathrm{T} 1$ patients and $72 \%$ at 5 years for T2 patients. They did not assess survival in T3 and T4 patients because of the small sample size. Additionally, a freedom from progression analysis was performed on patients with $\mathrm{T} 1$ and $\mathrm{T} 2$ disease who were treated with topical NM only (no additional therapies during the treatment course). At 20 years, $91 \%$ of T1 and $93 \%$ of T2 disease patients remained free from disease progression. This study also reported that patients who were continued on maintenance regimens of NM after initial treatment were found to have a longer response time compared to patients not on a maintenance regimen; however, the relapse rate was similar to those in the non-maintenance group when their maintenance therapy was discontinued. ${ }^{8}$

In 2005, a prospective study was conducted in France to evaluate 64 Stage IA-IIA MF patients treated with twiceweekly applications of $0.02 \%$ mechlorethamine solution in combination with topical steroids for 6 months. This study 
aimed to assess if decreasing the frequency of mechlorethamine application followed by application of topical steroids would be equal to or more effective in treating early stages of MF, in addition to decreasing cutaneous tolerance. CR to treatment was highest in Stage IA patients at $61 \%$, followed by $58 \%$ and $40 \%$ in Stage IB and Stage IIA patients, respectively. This study demonstrated similar efficacy of mechlorethamine with twice-weekly applications (58\% CR) compared to daily applications of NM as reported by Ramsay et al $(61 \% \mathrm{CR} \text { at } 2 \text { years })^{4}$ and $\mathrm{Kim}$ et al $(50 \% \mathrm{CR}) .{ }^{8}$ The time to CR noted in this study was significantly shorter than in previous studies referenced above. For Stage IA, Stage IB, and Stage IIA, CR was achieved after mean durations of 3.3, 3.8, and 3.0 months, respectively. This may reflect a synergistic effect with the combination of NM and topical steroids. ${ }^{9}$

More recent clinical trials have evaluated the safety of $0.02 \%$ mechlorethamine gel in comparison to $0.02 \%$ ointment-based preparations. A randomized controlled multicenter trial was conducted by Lessin et al in 2013 that demonstrated non-inferiority of gel-based mechlorethamine preparations in patients with Stage IA-IIA MF. Patients were randomized to either the gel or ointment arm and instructed to apply mechlorethamine once daily for up to 12 months until CR was achieved. The overall (complete and partial) response rate for the gel arm was $58.5 \%$ and for the ointment arm was $47.7 \%$. Compared to other studies that primarily report CR, the CR was $13.8 \%$ in the gel arm and $11.5 \%$ in the ointment arm (intent-to-treat population). On average, the time to response was 26 weeks ( 6.5 months) in the gel arm and 42 weeks (10.5) months in the ointment arm. In both the intent-to-treat population and the efficacy-evaluable population, the $\mathrm{CR}$ rates were between $11.5 \%$ and $18.9 \%$ in the combined gel and ointment arms. ${ }^{11}$ As mentioned above, Price et al reported a CR rate of $17.6 \%-27.3 \%$ in patients with Stage IA-IB treated with ointment-based mechlorethamine at concentrations of $0.01 \%{ }^{16}$ It is unclear as to why the $\mathrm{CR}$ rates for the gel and ointment preparations in this study are lower compared to the $\mathrm{CR}$ rates with aqueous-based preparations of mechlorethamine. The authors do note, however, that no additional concurrent therapy was used in patients with poor response, progressive, or unresponsive disease. The use of topical steroids, electron beam therapy, local radiation, or systemic treatments such as methotrexate or oral steroids was not permitted in this study. ${ }^{11}$

A Valchlor ${ }^{\circledR}$ extension trial by Kim et al with $0.04 \%$ mechlorethamine gel is currently underway for patients who did not receive a CR to the $0.02 \%$ mechlorethamine gel formulation in the previously mentioned study. Most recent data indicate $26.5 \%$ of patients have a confirmed response, with 6 complete responders and 20 partial responders. ${ }^{12}$

\section{Safety}

The safety of topical treatment with mechlorethamine is well established and documented in the studies presented in Table 3. Reported adverse events include immediate hypersensitivity reactions (ie, urticaria), DHR or ACD, irritant contact reactions, secondary malignancies including cutaneous melanoma and nonmelanoma skin cancers, and development of other primary malignancies such as colon cancer. Other cutaneous reactions include erythema, hyperpigmentation, pruritus, and skin irritation. ${ }^{11}$ DHRs are by far the most common adverse event or intolerance to aqueous NM treatment. ${ }^{4,5,8,17}$ Several studies have investigated methods to reduce the incidence of DHR by adjuvant treatment with topical steroids, decrease in frequency of treatments, and inducing tolerance to NM with incremental exposure. In addition, the ointment- and gel-based NM were developed to decrease contact hypersensitivity reactions while providing a more economically and user-friendly alternative to aqueous solutions. Urticarial type reactions or immediate hypersensitivity reactions are the most common reason for treatment termination in the initial stages of treatment. ${ }^{4,5}$ The concern for anaphylactic type reactions following urticaria is a common reason to terminate treatment in these patients. Additionally, studies are inconsistent with regard to the development of malignancy associated with the topical use of NM. As detailed below, most studies have not found a strong association between topical NM and cutaneous skin cancers.

\section{Delayed hypersensitivity reactions}

In the 1970s, Van Scott and Winters reported the incidence of DHR in 21 patients treated topically with NM at $28.6 \%$. One of the patients in the study developed a vesicular, irritant reaction that occurred during aggressive inpatient treatment. This study was conducted primarily to evaluate whether aggressive treatment with NM and DHRs enhance or alter the progression of MF. Patients were sensitized using varying applications of dissolved NM, intradermal injections, or whole body applications. Whole body applications were prepared by dissolving $10-40 \mathrm{mg}$ of $\mathrm{NM}$ in $30-40 \mathrm{~mL}$ of water, and it was further diluted if DHRs occurred. Six of 21 patients developed a DHR. The authors also found that patients who developed DHR achieved CR more rapidly, compared to patients who did not develop DHR. They noted resolution of both the DHR and clinical skin findings of MF in 2-3 weeks of the allergic reaction. ${ }^{17}$ 


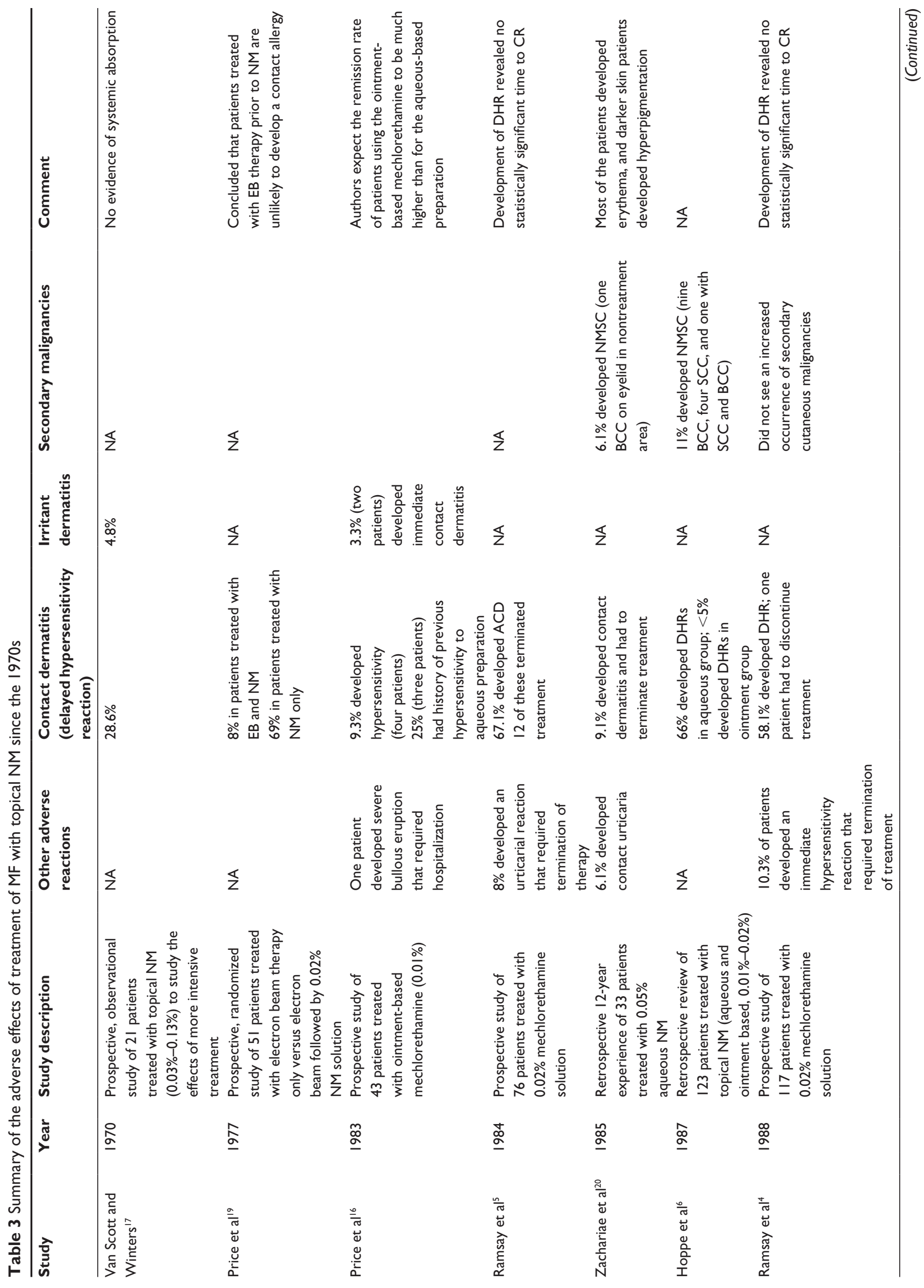




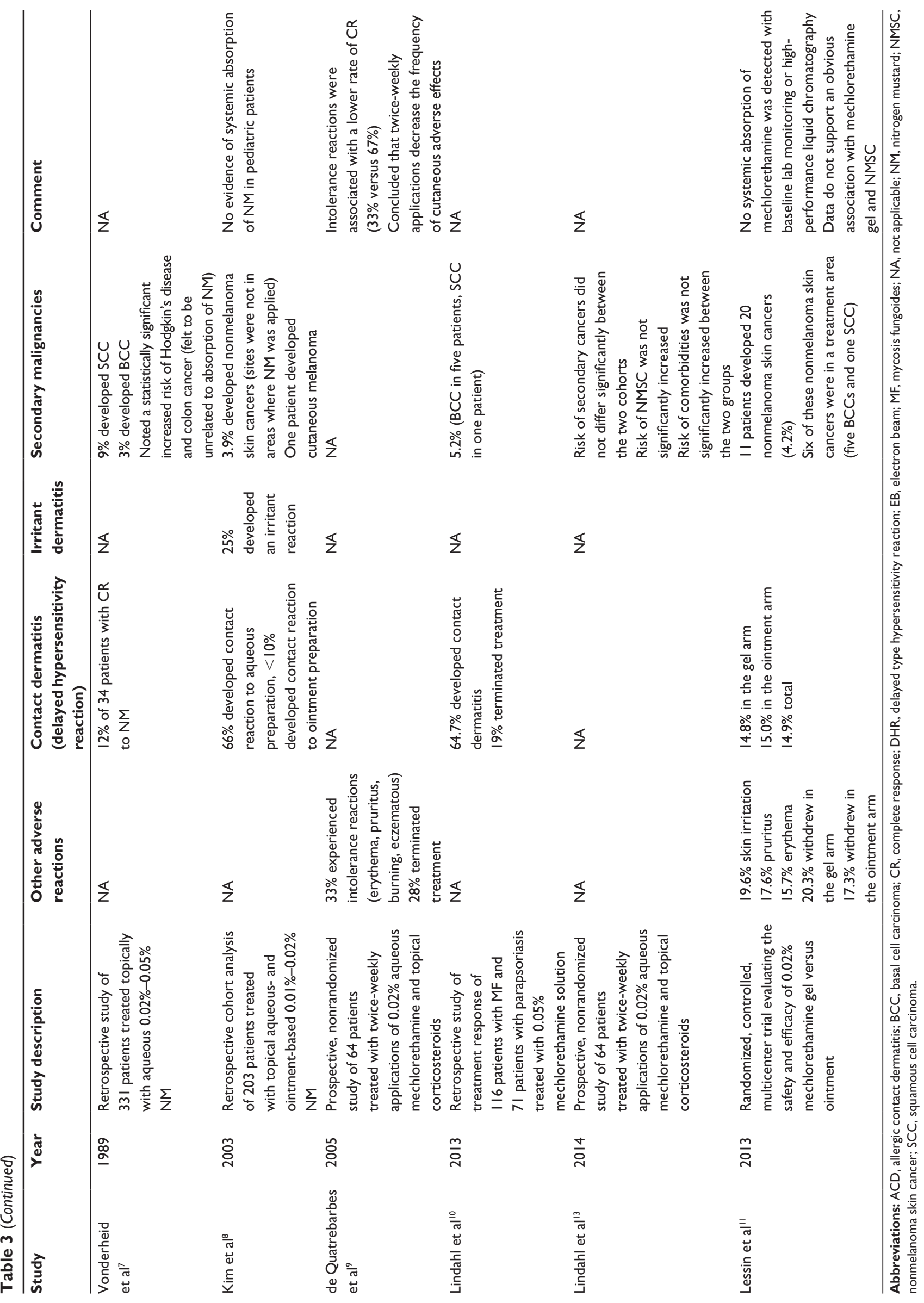


When evaluating patients treated with electron beam therapy plus NM and NM only patients, Price et al found marked difference in the development of DHR. They observed only an $8 \%$ incidence in contact dermatitis in patients treated with electron beam therapy prior to NM. In another subset of patients who only received topical $\mathrm{NM}$ and no irradiation, the incidence of contact dermatitis was $69 \%$. They hypothesized that electron beam therapy results in local immune suppression and, therefore, lessons the likelihood of a contact reaction to occur. ${ }^{19}$ At Stanford, Price et al further investigated the incidence of DHRs by evaluating ointment-based preparations of NM for treatment of MF. Mechlorethamine ointment at $0.01 \%$ was applied to the entire skin surface once per day and the concentration was increased to $0.02 \%$ for resistant disease. They found that 4 out of 43 patients (9.3\%) developed a DHR to ointment-based preparations. Only 3 of 12 patients with a previously documented DHR (25\%) developed a contact allergic reaction to the ointment-based preparations. The authors concluded that ointment-based preparations allow for preservation of mechlorethamine, preventing ionized breakdown products from eliciting a DHR. Compared to aqueous-based preparations, ointment-based formulations in this study used lower mechlorethamine, which may account for lower DHR. ${ }^{16}$

In 1988 , Ramsay et al observed that $58.1 \%$ of 117 patients treated with mechlorethamine solution developed a DHR. Only one patient had to discontinue treatment secondary to this reaction. These results reflect similar findings compared to the Ramsay et al 1984 study. In that study, 67.1\% of patients developed a DHR, with 12 patients requiring treatment termination. Mechlorethamine solution was prepared in a similar fashion to that described above by Van Scott et al, and patients were treated with topical steroids if they developed a DHR. Importantly, the authors found that there was no statistically significant difference in the probability of $\mathrm{CR}$ or the time to $\mathrm{CR}$ between patients who developed a DHR and those who did not. ${ }^{4}$ In 1984, Ramsay et al reported $69 \%$ of contact-sensitive patients achieved $\mathrm{CR}$ and $63.6 \%$ of non-contact-sensitive patients achieved $\mathrm{CR}$, and found no differences in time to $\mathrm{CR}$ between these groups. This is in contrast to the study referenced above by Van Scott and Winters, as they noted a faster time to CR in patients who developed a DHR. ${ }^{5,17}$ Vonderheid et al reported that $35 \%$ of patients with CR from topical NM developed a DHR/ACD. They observed no difference in the number of allergic reactions compared to patients with sustained remission of shorter duration. They do suggest that repeated DHRs promoted long-standing remission in some patients. ${ }^{7}$
Kim et al observed a slightly higher incidence of DHRs compared to Ramsay et al with aqueous-based preparations. They observed $66 \%$ of patients developed a DHR to aqueous preparations of NM $(0.01 \%-0.02 \%)$, while $<10 \%$ of DHRs were noted in patients who used ointment-based preparations of the same concentration. ${ }^{8}$ These findings are further supported by Hoppe et al who observed $66 \%$ of patients developed DHRs to aqueous-based preparations of $0.01 \%-0.02 \%$ mechlorethamine, while $<5 \%$ developed DHRs to ointment-based preparations of similar concentrations. ${ }^{6}$ Most of the patients in Kim et al's study were treated with ointment-based preparations and they reported a greater number of irritant contact reactions compared to allergic contact reactions. The authors noted that some patients with a rapid localized allergic reaction had earlier complete clearance. ${ }^{8}$ de Quatrebarbes et al also noted that CR was equivalent in patients with a mild reaction to NM compared to patients without cutaneous side effects (both $67 \%$ ). However, in patients who developed severe cutaneous reactions (extreme burning, pruritus, erythema, or eczema), the $\mathrm{CR}$ was one-third of that achieved in patients with mild cutaneous reactions and those who did not develop a cutaneous side effect. ${ }^{9}$

Lindahl et al reported the development of contact dermatitis or DHRs in $64.7 \%$ of patients treated with topical $0.05 \%$ NM solution, with termination of treatment in $19 \%$ secondary to intolerable side effects. ${ }^{10}$ While the frequency of contact dermatitis reported is similar to other studies, this study also used adjuvant treatment in $98.3 \%$ of patients, including topical steroids, psoralen + ultraviolet A, local radiation, and total skin electron beam therapy. ${ }^{4,5,8,10}$

A clinical trial was conducted in 2013 to assess the safety and efficacy of gel-based preparations of $0.02 \%$ mechlorethamine in comparison to $0.02 \%$ ointment-based preparations. Lessin et al reported $14.8 \%$ of patients in the gel arm and $15.0 \%$ of patients in the ointment arm experienced DHRs. This is substantially less than the reported incidence of DHRs with aqueous-based preparations. They noted the incidence of generalized skin irritation was statistically increased in the gel arm compared to the ointment arm. They also found that $61.7 \%$ of patients in the gel arm and $50.4 \%$ of patients in the ointment arm experienced at least one drug-related adverse event and $20.3 \%$ of patients in the gel arm and $17.3 \%$ of patients in the ointment arm withdrew from the trial. ${ }^{11}$

\section{Other cutaneous reactions}

Other cutaneous reactions reported in the literature include irritant contact dermatitis, severe bullous eruptions, urticarial reactions, and various intolerance reactions such as erythema 
with associated pruritus and burning. Van Scott and Winters ${ }^{17}$ reported an irritant contact dermatitis in $4.8 \%$ of patients, which is significantly lesser than the $25 \%$ incidence reported by Kim et al, ${ }^{8}$ but higher than the 3.3\% reported by Price et al. ${ }^{16}$ It should be noted that both Price et al and Kim et al used primarily ointment-based preparations. The irritant contact reactions reported by Kim et al were classified as mild and primarily located in thinner skin areas including the face or intertriginous areas. They also noted most patients improved, allowing for both continuation and escalation of therapy. ${ }^{8}$ Irritant like reactions were also reported by Lessin et al in patients treated with either gel or ointment preparations of mechlorethamine. In the gel arm, 25\% of patients experienced skin irritation, which was statistically significant compared to only $14 \%$ in ointment arm. Compared to other referenced studies, Lessin et al reported a higher incidence of irritant contact dermatitis in combined gel and ointment arms (19.6\%), as compared to ACD (14.9\%). This reflects that aqueous-based preparation of mechlorethamine is more likely to cause ACD/DHR and ointment- or gel-based preparations are more likely to have a higher incidence of irritant contact dermatitis. Lessin et al also found that $17.6 \%$ of patients in the combined gel and ointment arms developed pruritus, $15.7 \%$ developed erythema, 6.3\% developed skin hyperpigmentation, and $4.7 \%$ developed folliculitis. ${ }^{11}$

Price et al reported one patient in their 1983 study who developed a severe bullous eruption that required hospitalization secondary to intense swelling and inflammation of an entire limb. This patient achieved complete clearing after this reaction resolved. After 29 months, this patient had progression of disease, developing tumors and requiring additional therapeutic alternatives. ${ }^{16}$

Urticarial and other immediate hypersensitivity reactions are far less common compared to allergic contact or irritant contact reactions. Ramsay et al reported in 1984 that 8\% (6) patients and in 1988 that 10.3\% (12) patients developed an immediate urticarial reaction to NM that required treatment termination. These patients were felt to have a higher probability of anaphylaxis and started on alternative treatment modalities. ${ }^{5}$

\section{Secondary malignancies}

The development of secondary malignancies as a direct result of topical NM therapy is controversial. In 1988, Ramsay et al reported no observation of secondary malignancies in 117 patients after a median duration of 40 months. ${ }^{4}$ Kim et al reported $3.9 \%$ of patients treated with topical preparations of NM developed nonmelanoma skin cancers in sites that were not treated with NM. They also found one patient developed cutaneous melanoma; however, this patient was a Fitzpatrick Type I and had a prior history of nonmelanoma skin cancers. They also note that six of eight patients who developed nonmelanoma skin cancer were treated with other modalities known to cause an increase in cutaneous malignancies, including total skin electron beam therapy and phototherapy. The other two patients had a history of actinic damage, and skin cancers arose at sites that were not treated with NM. ${ }^{8}$ In 1989, Vonderheid et al reported an 8.6-fold increase in relative risk in the development of cutaneous squamous cell carcinoma and a 1.8-fold increase in the development of basal cell carcinoma in their series of 331 patients. In their study, $9 \%$ of patients developed squamous cell carcinoma and $3 \%$ of patients developed basal cell carcinoma. While these patients were treated with topical NM, there was also utilization of other treatment modalities such as electron beam therapy and UV radiation, both of which have carcinogenic capabilities. The authors felt that because development of skin cancers was noted in low sun exposure areas (genital) as well as in African American patients, the NM may have a direct carcinogenic effect. They also reported a statistically significant incidence of both Hodgkin's disease and colon cancer. This, however, was not thought to be caused by the absorption of $\mathrm{NM}$, and genetic and other pathologic mechanisms were felt to play a stronger role. ${ }^{7}$

Hoppe et al found the development of nonmelanoma skin cancer in $11 \%$ of patients treated topically with either NM ointment or aqueous preparations. Basal cell carcinoma was reported in 9 patients, squamous cell carcinoma in 4 patients, and 1 patient developed both squamous cell carcinoma and basal cell carcinoma. Of note, two of the four patients who developed squamous cell carcinoma, one of the scrotum and the other one on the arm, died from metastatic disease. Similar to other studies, most patients in the study had been treated with other topical modalities in addition to NM. ${ }^{6}$

In 2013 and 2014, Lindahl et al reported the development of secondary malignancies in patients using aqueous preparations of NM. In 2013, they reported secondary cutaneous malignancies in $5.2 \%$ (six patients) of patients with MF and 5.6\% (four patients) of patients with parapsoriasis treated topically with NM. Squamous cell carcinoma was found in one patient with MF and basal cell carcinoma in five patients with MF. It was noted that most patients in this group were treated with phototherapy or local radiation prior to treatment with NM and that most carcinomas were in sun-exposed areas. ${ }^{10}$ In 2014, Lindahl et al completed a 30 -year population-based cohort study to assess secondary 
cancer, comorbidities, and mortality associated with aqueous preparations of NM treatment. They found no significant increased risk of nonmelanoma skin cancers, melanomas, chronic lung disease, or lung cancer in the NM arm. ${ }^{13}$

Lessin et al observed the development of cutaneous malignancies in both the gel and ointment arms of NM during the 12-month clinical trial and throughout the 12-month follow-up period. They observed 20 nonmelanoma skin cancers in 11 patients ( 3 in gel arm and 8 in ointment arm). Of these, 10 were basal cell carcinoma, 9 were squamous cell carcinoma, and 1 was Merkel cell carcinoma. Five basal cell carcinomas and one squamous cell carcinoma occurred in treatment areas. The authors note a majority of these nonmelanoma skin cancers occurred in patients who had a history of skin cancers or had received prior phototherapy. ${ }^{11}$

\section{Systemic toxicity}

Several studies were designed to evaluate the possibility of systemic effects from absorption of topical NM. In 1970, Van Scott and Winters monitored white blood cell (WBC) counts on initiation of treatment and at intervals of 1-4 weeks depending on the frequency and dosing of NM. They did not find a decrease in the $\mathrm{WBC}$ or other systemic findings in these patients. The authors report similar findings in their 1973 study. ${ }^{15,17}$ In 2003, Kim et al evaluated systemic absorption of NM in pediatric patients. No evidence of systemic absorption was found, as indicated by normal WBC counts and chemistry panels completed every 2-3 months during treatment. ${ }^{8}$ In 2013, Lessin et al were the first to perform highperformance liquid chromatography serum assays to assess for systemic absorption of mechlorethamine in both the gel and ointment preparations. A subset of patients was evaluated several hours after the initial application and again at 4 weeks. The authors found no evidence of systemic absorption by clinical laboratory monitoring (baseline, months 4 , 8,12 ) or detection of mechlorethamine by high-performance liquid chromatography. ${ }^{11}$

\section{Discussion}

NM or mechlorethamine has evolved as a treatment for early-stage MF since the 1950s in terms of primary vehicle, as well as treatment frequency and duration. The safety and efficacy is well documented in the numerous aforementioned studies.

The efficacy data with NM aqueous solutions is well established, but variability in treatment application, frequency, duration, and additional therapies makes these studies difficult to compare. Approximately 51\%-84\% of T1 patients achieve CR with aqueous topical NM and $34 \%-62.2 \%$ of T2 patients achieve CR with topical NM. Ointment- and gel-based preparations have, in general, shown lower rates of CR. In the recent non-inferiority study, Lessin et al reported similar overall response rates, but with notably lower CRs. It should be emphasized that the majority of studies in this review used adjuvant treatment modalities concurrently with topical NM. Lessin et al used NM only as a single agent and did not permit the use of other therapies, especially topical steroids or systemic treatments. They also excluded patients who had used topical NM within the last 2 years and those who had received radiation therapy within the last 1 year. This may explain, in part, the reduction in CR. Further studies will need to be developed to assess the efficacy of mechlorethamine gel in combination with additional treatment modalities and at higher concentrations.

In the 1980s, ointment preparations became a more common method of application as they were more economical and resulted in a lower incidence of hypersensitivity reactions, compared to aqueous-based preparations. More recently, the gel preparation has become widely utilized. As mentioned above, Price et al hypothesized that ointment-based preparations prevent the ionized breakdown products of mechlorethamine which are thought to be responsible for the DHR. ${ }^{16}$ The decreased incidence of DHRs in ointment and gel preparations has been replaced with increasing irritant reactions, pruritus, and erythema, when compared to aqueousbased preparations. Overall, around a third of patients are reported to have developed irritant dermatitis, urticaria, DHRs, or other immediate hypersensitivity reactions, some of which have resulted in termination of treatment. ${ }^{4,9,11}$ While efforts have been made to decrease adverse events through alteration of vehicle, application schedule, and concomitant use of topical corticosteroids, we continue to encounter these events in our patients today. It is important to note that all studies reviewed in this report found no evidence of systemic absorption of NM as evidenced by normal white blood counts, normal chemistry panels, and no detection of mechlorethamine by high-performance liquid chromatography at varying treatment intervals.

Secondary malignancies have been reported to occur between $3.9 \%$ and $12 \%$ in patients treated with aqueous-, ointment-, or gel-based preparations of NM. Most studies comment that there is no increased risk of secondary malignancies as a result of topical NM, and the studies frequently allowed for alternative treatment modalities including localized radiation and phototherapy, both of which are known carcinogens. Additionally, several studies recognize the 
development of nonmelanoma skin cancers in prior sundamaged skin or sun-exposed areas. Vonderheid et al did observe development of skin cancers in atypical patient populations and non-sun-exposed areas, postulating that NM may have carcinogenic potential. ${ }^{7}$ Overall, the role of $\mathrm{NM}$ in the development of secondary cancers has not been fully established, but repeated application of an alkylating chemotherapeutic agent could potentially increase the risk.

NM has a long history in the treatment of CTCL and specifically in the cutaneous lesions of MF. Throughout the last seven decades, multiple publications have demonstrated its efficacy as well as its side effect profile, and NM will continue to serve our patients.

\section{Disclosure}

Dr McGirt is a consultant for Actelion and Mallinkrodt. The authors report no other conflicts of interest in this work.

\section{References}

1. Elston D, Ferringer T, Ko CJ, High WA, DiCaudo DJ. Dermatopathology. Philadelphia: Elsevier; 2014.

2. Olsen E, Vonderheid E, Pimpinelli N, et al; ISCL/EORTC. Revisions to the staging and classification of mycosis fungoides and Sezary syndrome: a proposal of the International Society for Cutaneous Lymphomas (ISCL) and the cutaneous lymphoma task force of the European Organization of Research and Treatment of Cancer (EORTC). Blood. 2007;110(6):1713-1722.

3. Haserick J, Richardson JH, Grant DJ. Remission of lesions in mycosis fungoides following topical application of nitrogen mustard: a case report. Cleve Clin Jf Med. 1959:144-147.

4. Ramsay DL, Halperin PS, Zeleniuch-Jacquotte A. Topical mechlorethamine therapy for early stage mycosis fungoides. J Am Acad Dermatol. 1988;19(4):684-691.

5. Ramsay DL, Parnes RE, Dubin N. Response of mycosis fungoides to topical chemotherapy with mechlorethamine. Arch Dermatol. 1984; 120(12):1585-1590.

6. Hoppe RT, Abel EA, Deneau DG, Price NM. Mycosis fungoides: management with topical nitrogen mustard. J Clin Oncol. 1987;5(11): 1796-1803.
7. Vonderheid EC, Tan ET, Kantor AF, Shrager L, Micaily B, Van Scott EJ. Long-term efficacy, curative potential, and carcinogenicity of topical mechlorethamine chemotherapy in cutaneous $\mathrm{T}$ cell lymphoma. $J \mathrm{Am}$ Acad Dermatol. 1989;20(3):416-428.

8. Kim YH, Martinez G, Varghese A, Hoppe RT. Topical nitrogen mustard in the management of mycosis fungoides: update of the Stanford experience. Arch Dermatol. 2003;139(2):165-173.

9. de Quatrebarbes J, Esteve E, Bagot M, et al. Treatment of early-stage mycosis fungoides with twice-weekly applications of mechlorethamine and topical corticosteroids: a prospective study. Arch Dermatol. 2005; 141(9):1117-1120.

10. Lindahl LM, Fenger-Gron M, Iversen L. Topical nitrogen mustard therapy in patients with mycosis fungoides or parapsoriasis. J Eur Acad Dermatol Venereol. 2013;27(2):163-168.

11. Lessin SR, Duvic M, Guitart J, et al. Topical chemotherapy in cutaneous T-cell lymphoma: positive results of a randomized, controlled, multicenter trial testing the efficacy and safety of a novel mechlorethamine, $0.02 \%$, gel in mycosis fungoides. JAMA Dermatol. 2013;149(1): $25-32$.

12. Talpur R, Venkatarajan S, Duvic M. Mechlorethamine gel for the topical treatment of stage IA and IB mycosis fungoides-type cutaneous T-cell lymphoma. Expert Rev Clin Pharmacol. 2014;7(5):591-597.

13. Lindahl LM, Fenger-Gron M, Iversen L. Secondary cancers, comorbidities and mortality associated with nitrogen mustard therapy in patients with mycosis fungoides: a 30-year population-based cohort study. Br J Dermatol. 2014;170(3):699-704.

14. Price NM, Constantine VS, Hoppe RT, Fuks ZY, Farber EM. Topical mechlorethamine therapy for mycosis fungoides. Br J Dermatol. 1977; 97(5):547-550.

15. Van Scott EJ, Kalmanson JD. Complete remissions of mycosis fungoides lymphoma induced by topical nitrogen mustard (HN2). Control of delayed hypersensitivity to HN2 by desensitization and by induction of specific immunologic tolerance. Cancer. 1973;32(1):18-30.

16. Price NM, Hoppe RT, Deneau DG. Ointment-based mechlorethamine treatment for mycosis fungoides. Cancer. 1983;52(12):2214-2219.

17. Van Scott EJ, Winters PL. Responses of mycosis fungoides to intensive external treatment with nitrogen mustard. Arch Dermatol. 1970; 102(5):507-514.

18. Apisarnthanarax N, Talpur R, Duvic M. Treatment of cutaneous T cell lymphoma: current status and future directions. Am J Clin Dermatol. 2002;3(3):193-215.

19. Price NM, Hoppe RT, Constantine VS, Fuks ZY, Farber EM. The treatment of mycosis fungoides: adjuvant topical mechlorethamine after electron beam therapy. Cancer. 1977;40(6):2851-2853.

20. Zachariae H, Thestrup-Pedersen K, Sogaard H. Topical nitrogen mustard in early mycosis fungoides. A 12-year experience. Acta Derm Venereol. 1985;65(1):53-58.
Drug Design, Development and Therapy

\section{Publish your work in this journal}

Drug Design, Development and Therapy is an international, peerreviewed open-access journal that spans the spectrum of drug design and development through to clinical applications. Clinical outcomes, patient safety, and programs for the development and effective, safe, and sustained use of medicines are the features of the journal, which

\section{Dovepress}

has also been accepted for indexing on PubMed Central. The manuscript management system is completely online and includes a very quick and fair peer-review system, which is all easy to use. Visit http://www.dovepress.com/testimonials.php to read real quotes from published authors. 